\section{DEFORMITIES OF THE NASAL SEPTUM.}

\author{
BY J. H. McCASSY, M.A., M.D. \\ DAYTON, OHIO.
}

But little success in the treatment of diseases of the upper respiratory passages and the ear can be attained while occlusion or partial occlusion of one or both nostrils exists. It is essential, therefore, that deflections of the septum nasi and other intranasal abnormalities be removed at the outset, so that the ingoing current of air may pass through the nasal chambers and be properly warmed and moistened therein. Physicians will not question the soundness of these conclusions, yet they too frequently let patients thus afflicted go the "rounds" without offering them advice and suggestions as to the necessity of primarily removing intranasal abnormalities for the ultimate success of the management of these cases. Mouth breathing, headache, epistaxis, sneezing, frontal neuralgic pains, insomnia and total or partial deafness are common symptoms of nasal obstruction. Chronic nasal catarrh and septal deformities go hand in hand.

Classification-1, osseous; 2, cartilaginous; 3, osseo-cartilaginous (Jarvis). This is the best and most natural classification. The seat of the deformity is found in the cartilaginous septum in twothirds of the cases.

Causation-Civilization seems to be a factor of nasal deformity, for the American and European races are prone to them. Collier says about 80 per cent. of the savages are exempt from them. During early life falls or blows on the nose or rough wiping of it, sets up a low grade of inflammation-the perichondrium is over-nourished, new cells are deposited and the cartilage within its unyielding framework bulges to one side from excessive growth. Usually considerable thickening occurs at the eminence forming enchondroma and at the junction of the cartilage with the bone, bony deposits occur forming exostosis.

Cuse 1.-Deflection of the cartilaginous septum with ecchon. droma. Mr. M., aged 30 years suffered for many years with chronic nasal catarrh, pharyngitis and deafness. $\mathrm{He}$ complained of fullness in his head, sneezing, frontal neuralgic pain and insomnia. Examination revealed deviation to the left and great thickening of the cartilaginous septum. The ecchondroma was situated about half an inch back from the nasal outlet. This exerted considerable pressure against the outer wall of the left nostril, giving rise to such reflex symp. toms as sneezing, headache, frontal neuralgic pain and lachrymation.

An 8 per cent. solution of cocain was applied for eight minutes, which produced anesthesia and shrunk the spongy tissue enabling the writer to view the contour of the parts. A scalpel with a narrow blade was used to excise the redundant tissue. Commencing at the floor of the nostril, the incision was carried inward three-sixteenths of an inch, then curved upward about half an inch. An assistant kept a close watch in the other nostril to warn against cutting through the septum. This precaution precludes the possibility of such a mishap. This operation was completed in a few seconds. A full view of the parts being cut is the safeguard in this operation. Delay will result in obscuration of the parts from hemorrhage. A sharp, narrow-bladed knife will remove cartilaginous outgrowths readily. It being impossible to determine beforehand the presence or absence of bony tissue the writer usually begins the operation with the knife, only using the saw and alligator forceps when absolutely necessary. A 12 per cent. solution of the nitrate of silver was brushed on the cut surface to aid in arresting hemorrhage. In a few minutes bleeding ceased. The nostril was then washed out with Dobell's solution. The patient was directed to blow the clote out. The nostril was then packed with cotton saturated with carbolized vaselin. This dressing was used for two weeks, being changed once or twice daily for the purpose of cleansing. As a result of this operation a good patulous nostril was secured and septum straightened considerably. The naso-pharyngeal catarrh yielded kindly to astringents of nitrate of silver and chlorid of zinc and alkaiin and albolene sprays. The reflex symptoms complained of soon disappeared.

Case 2.- Exostosis and ecchondroma with deflection of the septum. Mr. S., aged 45 years, a bookkeeper, was referred to the writer by Dr. Ensey in July, 1896, for naso-pharyngitis, and on-coming deafness. Examination showed that the septum was but slightly deviated to the right, but a large exostosis and ecchondroma, located about half an inch back from the nasal outlet, occupied the floor and septal side of right nostril, shutting off the passage of air. This precluded the possibility of using the Eustachian catheter in the right ear.

Under cocain anesthesia, with the scalpel an elliptical portion of the mucous membrane was removed from the eminence of the outgrowth. A mastoid curette was used to separate and push back about a quarter of an inch the mucous membrane toward the base of the tumor. The knife failing to cut the exostosis the saw was used for a while, but owing to the shape of the tumor the saw could only be used to outline the cut both above and below. Then the alligator cutting forceps was used and the growth cut or twisted off in five pieces. Hemorrhage was pretty free for a few minutes. The parts were cleansed with Dobell's solution and the mucous membrane was coapted as nearly as possible without stitches. The nostril was packed with cotton saturated with carbolized vaselin. The after-treatment was the same as in Case 1. Healing was uneventful; a good patulous nostril was secured and the catarrhal symptoms soon diminished.

Case 3.-Deflection of the nasal septum without exostosis or ecchondroma. Mr. T., aged 35 years, was referred to the writer by his family physician in March, 1893. He was a mouth-breather and had copious secretions from his nasopharyn $x$ and from his right nostril. As long as he can remember he has suffered with headache, sneezing and frontal neuralgic pains. $\mathrm{He}$ said he had taken a barrel of medicine without getting any relief from his trouble. Examination revealed an enormous hypertrophy of his right inferior turbinal and the cartilaginous septum was curved to the left nostril so as to press against the outer wall of the left nostril. completely occluding it.

The hypertrophy was removed by two linear incisions to the bone drawn from the back to the front with the cautery knife heated to a white heat. This was done under cocain anesthesia at two sittings.

Then under cocain anesthesia an operation was made in the septum with an old cataract knife. An incision an inch long was made borizontally from before backward; another incision one-eighth of an inch in the middle was made below the first one, which tapered to nothing at either end. This permitted an elliptical portion of the cartilage and mucous mem brane to be removed. By means of two vertical incisions a similar but shorter elliptical portion of the septum was removed. This made a stellate opening in the cartilaginous septum. (Max Wocher of Cincinnati had made to the writer's order a clamp with the blades over an inch long by threefourths of an inch wide. When the blades were clasped firmly they were held in place by a set of screws, each blade had a hole in the center of it one line in diameter for the purpose of drainage.) After the hemorrhage had ceased the parts were cleaned and the clamp applied firmly by a pair of long forceps. The firm pressure straightened the septum and coapted the cut edges nearly. The clamp was worn for three days, being removed daily and the parts cleansed. A few doses of morphin were given to allay the pain and uncomfortable feeling arising from the pressure of the clamp. At the end of the third day the clamp was displaced by a perforated hollow rubber splint which was worn for two weeks, being removed daily for cleansing purposes. Healing occurred without interruption. The case remained under treatment for three months, during which time the naso-pharynx was brushed every second day with a 2 to an 8 per cent. solution of nitrate of silver and sprayed with Dobell's solution, each treatment being concluded with a spray of albolene containing a grain to the ounce of both carbolic acid and menthol. At the end of this time the naso-pharyngeal catarrh was practically cured and the headache and neuralgia had disappeared.

These cases are not reported here for uniqueness but because they represent fairly well the prevailing types of deformities of the cartilaginous nasal septum, not including spurs. The line of treatment adopted is simple, but successful. No clamp or reasonable amount of pressure will straighten simple deflection 
of the septum while too much tissue remains in it. The redundant cartilage or bone must be removed. Fortunately simple deflection of the septum is rare. Not many cases will require the operation described in Case 3. In the great bulk of cases if the redundant cartilage or bone is pared off and the septum thinned thereby, the tissue in healing contracts and helps to straighten the septum and a good breathing space will be secured. The writer usually adopts the simpler operation first, leaving the clamp operation as a last resort. Indeed it is difficult to get patients to undergo the clamp operation. In cases having no manifestation of syphilis or scrofula little fear need be entertained for perforation or ulceration; delayed healing may occur but not the former. It is not necessary to be too penurious about the loss of a little mucous membrane. It will readily reform and produce a healthy secreting surface.

32 West Fifth Street.

\section{FOLLICULAR OR CROUPOUS TONSILLITIS.} Abstract of a paper read before the Falls Cicy Medical Society,

BY P. RICHARD TAYLOR, M.D.

Professor Materia Medica and Therapeutics, Clinical Lecturer on Diseases of the Eye, Ear, Nose and Throat, and Dean of the Faculty of the Hospital College of Medicine; Member of Kentucky Medical society, etc. LOUISVILLE, KY.

Follicular tonsillitis usually begins with a temperature of 102 to 104 degrees, accompanied by chills and rigors, a full bounding pulse, throbbing headache, aching of the bones of the extremities and loss of appetite. The tonsillar symptoms do not become prominent until six to twelve hours later. Fulness is felt on deglutition, and a sensation as though a jagged foreign body was projecting from the tonsil; the anterior and posterior pillars and tonsil will be swollen, the follicles will be larger than normal and filled with a gray exudate and coagulated lymph.

At the end of twenty-four hours, the throat symptoms will be markedly exaggerated; the inflammation will have extended to the pharynx, and probably to the larynx; the soft palate will be edematous, the uvula elongated, and the feeling of fulness and stiffness of the muscles of the throat will be increased. There is dryness of the pharynx and greater swelling and edema of the pillars. The deposits in the follicles have become profuse and may be continuous over the surface of the tonsil. The pain has greatly increased and is continuous, being extreme in deglutition; the lymphatics about the neck are usually involved. The attack lasts from seven to ten days, but may be prolonged by the infection attacking first only one tonsil and later the other.

Cause.-The predisposing cause is enlarged tonsils with open crypts and follicles with ragged edges. That it is due to a specific organism is unquestioned. A number of organisms have been found in the exu. date, but just which one produces the disease has not been determined.

Treatment. - The temperature, throbbing pain in the head and aching of the limbs can best be controlled with quinin and some of the coal tar derivatives, preferably phenacetin. I get the best results from $2 \frac{1}{2}$ grains each administered every two hours until distressing symptoms are relieved. Gargles of very hot water will increase the patient's comfort and lessen edema.

The tonsils, being the seat of the disease as well as the point of infection, demand the greatest attention. The exudate upon the surface of the tonsil can be easily wiped away with a probe armed with dry cotton. Usually, any attempt to dislodge it with the probe from the follicles results in its being pushed deeper into them.

For the last eighteen months, I have adopted a method which renders it easy to thoroughly cleanse the tonsil and which shortens the duration of the attack, and in many cases, seen early, completely aborts it. I use a blow pipe made of soft silver about seven inches long, curved at the smaller end, resembling a long, curved Eustachian catheter; in fact, the catheter can be used very nicely as a substitute. After wiping away with the probe and cotton all exudate from the surface of the tonsil, I then attach this blow pipe to the cut-off from my air receiver, just as I would a spray tube. The curved end should be placed about one inch from the tonsil, the air turned on at about twenty pounds pressure, and the current of air passed rapidly from follicle to follicle, which will completely remove all accumulations.

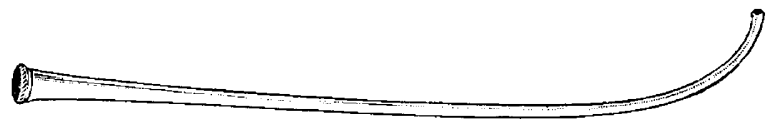

The follicles are then washed with a solution of bichlorid, 1 to 3,000, with an angular Saas spray tube held very close to the follicles, after which the tonsil and sometimes the surrounding mucous membrane is painted with a solution of nitrate of silver, 40 to 60 grains to the ounce. The application of the silver solution will have a decided anesthetic effect and your patient will experience great relief. Other applications may be substituted for the silver solution, but I have found it serves the purpose best. This treatment should be repeated twice or thrice daily according to the severity of the attack. Iodin should be avoided in all acute stages.

To abort this disease the case must be seen during the first twenty-four hours of the attack, the earlier the better. Even when seen too late to abort the attack, this treatment materially lessens the severity.

Incidentally, I have successfully used the same blow pipe to remove soft accumulations and small foreign bodies from the ear.

\section{THE DIAPHRAGM AND CENTRUM TENDINEUM.}

WITH TEN ORIGINAL ILLUSTRATIONS BY THE AUTHOR.

BY BYRON ROBINSON, B.S., M.D.

PROFESSOR OF GYNECOLOGY IN POST-GRADUATE SCHOOL AND POLICLINIC. CHICAGO.

(Concluded from page 20\%.)

The membrana limitans is a very fine, thin connective tissue layer on which the endothelia rest. It if a finely granular, or better, a finely fibrillar or striped membrane. It contains no cells. It has a watery or glass-like transparency, which is plainly observed when the endothelia are fallen off or very lightly brushed off. As Bizzozero and Salvioli have shown, the membrane is not exactly alike over all the parts of the peritoneum, but is perforated over the diaphragm. They say that the perforations of the membrana limitans are situated on the zona tendinea, and the zona peritendinea of the diaphragm. Bizzozero and Salvioli assert that the pores of the membrana limitans have a diameter from four to sixteen $\mathrm{mm}$. and 